\title{
An Analysis of the Pathogenic Genes and Mutation Sites of Macrodactyly
}

\author{
Jian-Feng Li', Guang-Lei Tian², Hui Pan³, Wen-Tong Zhang', Da-Cun Li', Jing-Da Liu', Liang Zhao', \\ Hai-Lei Li \\ 'Department of Hand Surgery, Beijing Shunyi District Hospital; Shunyi Teaching Hospital of Capital Medical University, Beijing, I0I300, People's \\ Republic of China; ${ }^{2}$ Department of Hand Surgery, Beijing Jishuitan Hospital; Peking University Fourth School of Clinical Medicine, Beijing, I00035, \\ People's Republic of China; ${ }^{3}$ Department of Pathology, Beijing Shunyi District Hospital; Shunyi Teaching Hospital of Capital Medical University, Beijing, \\ 101300, People's Republic of China
}

Correspondence: Jian-Feng Li, Department of Hand Surgery, Beijing Shunyi District Hospital; Shunyi Teaching Hospital of Capital Medical University, No. 3 Guangming South Street, Shunyi District, Beijing, 101300, People's Republic of China, Tel +8610 69423220, Email drlijf27@I26.com

\begin{abstract}
Objective: This study aimed to explore the pathogenic genes and mutation sites of macrodactyly.
Methods: Whole-exome sequencing was performed on the pathological tissue and peripheral blood of 12 patients with macrodactyly who were operated in our hospital between June 2018 and May 2020. In order to conduct comprehensive bioinformatics analysis and screen the pathogenic genes of macrodactyly, the patients were divided into four groups: macrodactyly of finger group, macrodactyly of foot group, macrodactyly and syndactyly of finger group, and macrodactyly and syndactyly of foot group. The results of the wholeexome sequencing were verified using Sanger sequencing in order to clarify the pathogenic genes and mutation sites of macrodactyly, and immunohistochemical analysis of the protein signaling pathways encoded by the pathogenic genes was performed to observe the protein expression and further verify the mutant genes.

Results: In the comprehensive bioinformatics analysis and Sanger verification of the whole-exome sequencing, the PIK3CA gene mutation was screened as the pathogenic gene of macrodactyly. The mutation sites were identified as the p.E542K (c.G1624A) and p. E545K (c.G1633A) sites of exon10 and the p.H1047R (c.A3140G) and p.G1049R (c.G3145C) sites of exon21. Among these, the p. G1049R (c.G3145C) locus was found in macrodactyly for the first time. The mutation of the PIK3CA gene was also found to lead to increased expression of serine-threonine kinase (AKT) in adipocytes in the PI3K-AKT-mTOR signaling pathway.

Conclusion: Mutation of the PIK3CA gene leads to the enhancement of the PI3K-AKT-mTOR signaling pathway, which is the cause of macrodactyly. There is also some diversity in PIK3CA gene mutation sites.
\end{abstract}

Keywords: hand deformity, foot deformity, macrodactyly, PIK3CA gene

\section{Introduction}

Congenital macrodactyly is a rare non-hereditary congenital malformation, often involving one or more fingers or toes and manifesting as diffuse, asymmetric enlargement and continuous growth of the palms or soles. It can cause serious impairment of limb appearance and function. The incidence of macrodactyly in newborns is about 1 in 100,000, accounting for approximately $1 \%$ of congenital deformities of limbs. ${ }^{1}$ Most of these patients are sporadic cases, and excessive adipose tissue hyperplasia and nerve-fiber fat infiltration are the main pathological changes. ${ }^{2}$ Currently, the treatment of macrodactyly mainly relies on surgical procedures for soft-tissue reduction and osteotomy to improve limb appearance, but the results are not yet completely satisfactory. ${ }^{3-5}$

Previous studies have revealed that PIK3CA-related overgrowth spectrum has part of the phenotype of macrodactyly, ${ }^{6,7}$ in the present study, whole-exome sequencing (WES) was performed on the diseased tissue and peripheral blood of 12 patients with macrodactyly who were operated on in our hospital between June 2018 and May 2020. In order to conduct comprehensive bioinformatics analysis and screen the pathogenic genes of macrodactyly, the patients were divided into four groups: macrodactyly of finger group, macrodactyly of foot group, macrodactyly and 
syndactyly of finger group, and macrodactyly and syndactyly of foot group. The results of the WES were verified using Sanger sequencing in order to identify the mutation sites, and immunohistochemical analysis of the protein signaling pathways encoded by the pathogenic genes was performed to observe the protein expression and further verify the mutant genes, thereby providing a theoretical basis for the pathogenesis of macrodactyly.

\section{Subjects and Methods}

\section{General Data}

Of the 12 patients enrolled in the study, 6 were males and 6 were females, and the average age was 3.5 years (range: 1-13 years). All 12 patients are Chinese and were born in China. All the 12 patients are not familial macrodactylyThe patients were divided into four groups according to the presence of macrodactyly (finger or toe), and the presence of syndactyly (finger or toe): macrodactyly of finger group (3 cases), macrodactyly of foot group (5 cases), macrodactyly and syndactyly of finger group ( 2 cases), and macrodactyly and syndactyly of foot group ( 2 cases). Peripheral blood samples were available in 9 of the 12 patients. The general information and grouping of the patients are shown in Table 1 and Figure 1.

\section{Inclusion and Exclusion Criteria}

All enrolled patients were clinically diagnosed with simple macrodactyly. Patients with congenital limb hypertrophies caused by congenital lymphatic and vascular malformations, such as CLOVES (congenital lipomatous overgrowth, vascular malformations, epidermal nevi, and skeletal/scoliosis and spinal abnormalities) syndrome, Klippel-Trenaunay syndrome, and Proteus syndrome, were excluded.

\section{Specimen Acquisition}

During each patient's surgical procedure (Figure 2), $0.5 \mathrm{~g}$ of pathological tissue (fat tissue) was resected and $1 \mathrm{~mL}$ of the peripheral blood (EDTA anticoagulation) was taken. The samples were labeled accordingly, before being transported on dry ice and stored at a temperature of $-80^{\circ} \mathrm{C}$.

Prior to specimen acquisition, the consent of the patients and their legal guardians was obtained, and an informed consent form was signed. The specimens taken for the study were discarded pathological tissues that were resected during surgery, so the acquisition of the specimens would not harm the patient and the confidentiality of the patient's genetic information was assured. The study was approved by the ethics committee of our hospital.

Table I Grouping and General Situation of 12 Children with Giant Finger (Toe) Disease

\begin{tabular}{|l|l|l|l|l|l|l|}
\hline No. & Group & Gender & Age (Year) & Finger (Toe) & Tissue Specimens & Blood Sample \\
\hline I & Pure macrodactyly & Male & 4 & Right I, 2 & Yes & Yes \\
2 & Pure macrodactyly & Female & 2 & Left I, 2,3 & Yes & No \\
3 & Pure macrodactyly & Male & 5 & Right 3, Palm & Yes & No \\
4 & Pure macrodactyly & Male & 1 & Left I, 2 & Yes & Yes \\
5 & Pure macrodactyly & Female & 13 & Right I, 2,3,4 & Yes & Yes \\
6 & Pure macrodactyly & Male & 6 & Right I, 2 & Yes & Yes \\
7 & Pure macrodactyly & Male & 3 & Right 2,3 & Yes & Yes \\
8 & Pure macrodactyly & Female & 8 & Left 2,3 & Yes & Yes \\
9 & Macrodactyly and syndactylia & Male & 2 & Right 3,4 & Yes & No \\
10 & Macrodactyly and syndactylia & Female & 1 & Left 2,3 & Yes & Yes \\
11 & Macrodactyly and syndactylia & Female & 4 & Right 2,3 & Yes & Yes \\
12 & Macrodactyly and syndactylia & Female & 1 & Right 2,3 & \\
\hline
\end{tabular}


A

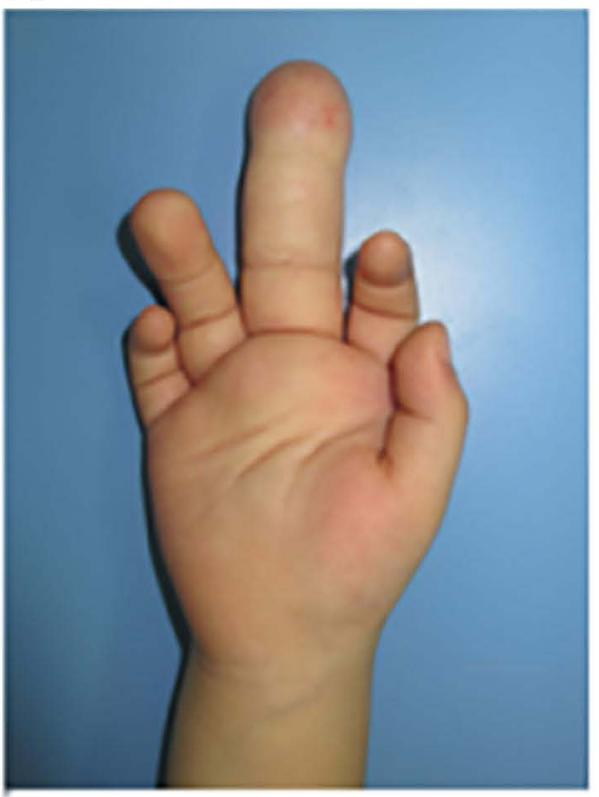

C

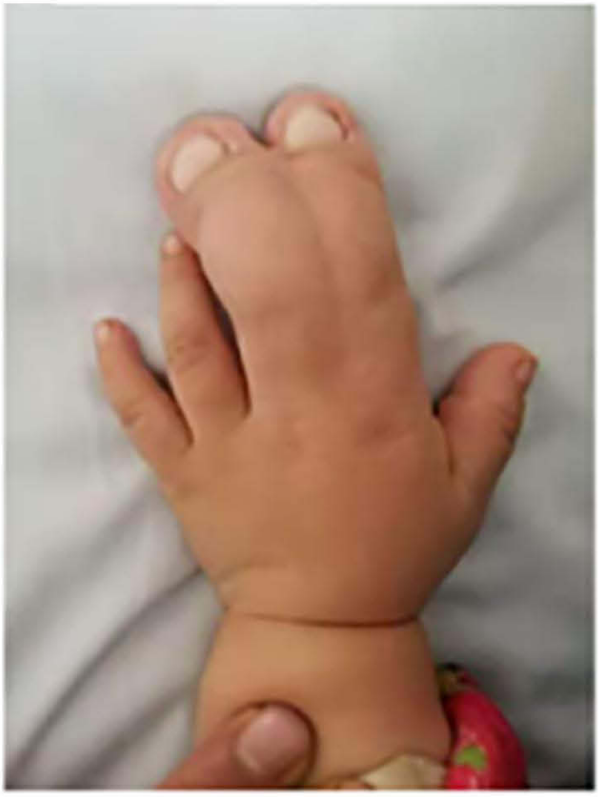

B

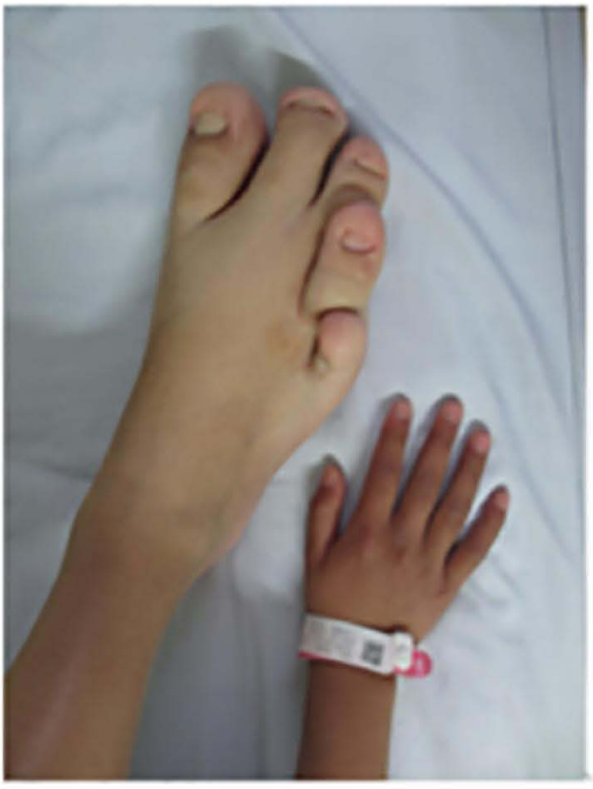

D

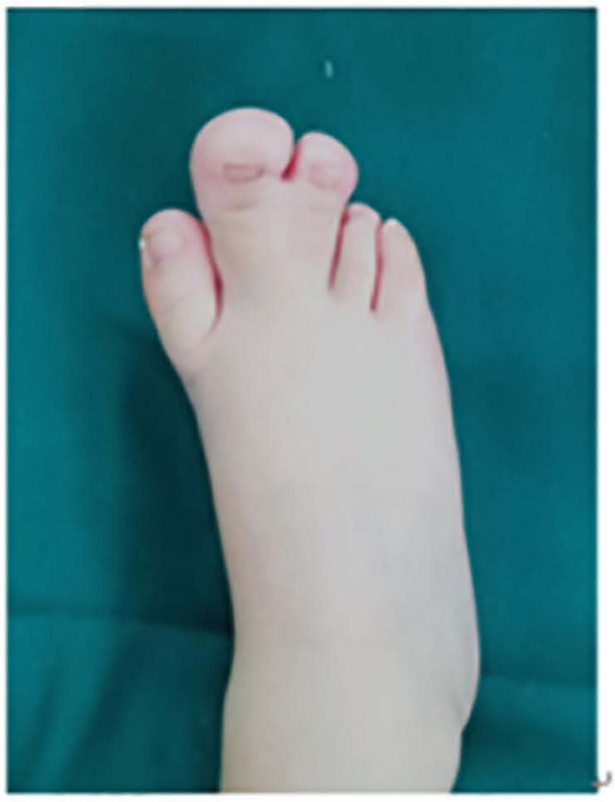

Figure I Clinical features of macrodactyly ((A) macrodactyly of finger; (B) macrodactyly of foot; (C) macrodactyly and syndactyly of finger; (D) macrodactyly and syndactyly of foot).

\section{The WES Process DNA Sample Testing}

Agarose gel electrophoresis was performed to analyze the degree of DNA degradation and identify any RNA and protein contamination. Qubit 3.0 (Life Invitrogen, USA) was used to accurately quantify DNA concentration.

\section{Library Building and Capture}

The genomic DNA was randomly broken into fragments of 180-280 bp in length using a Covaris ultrasonic disruptor (Covaris, USA). After terminal repair and A-tailing, linkers were connected to both ends of the fragment to build a DNA library. The library building and capture experiment was performed using an Agilent SureSelect Human All Exon V6 kit 


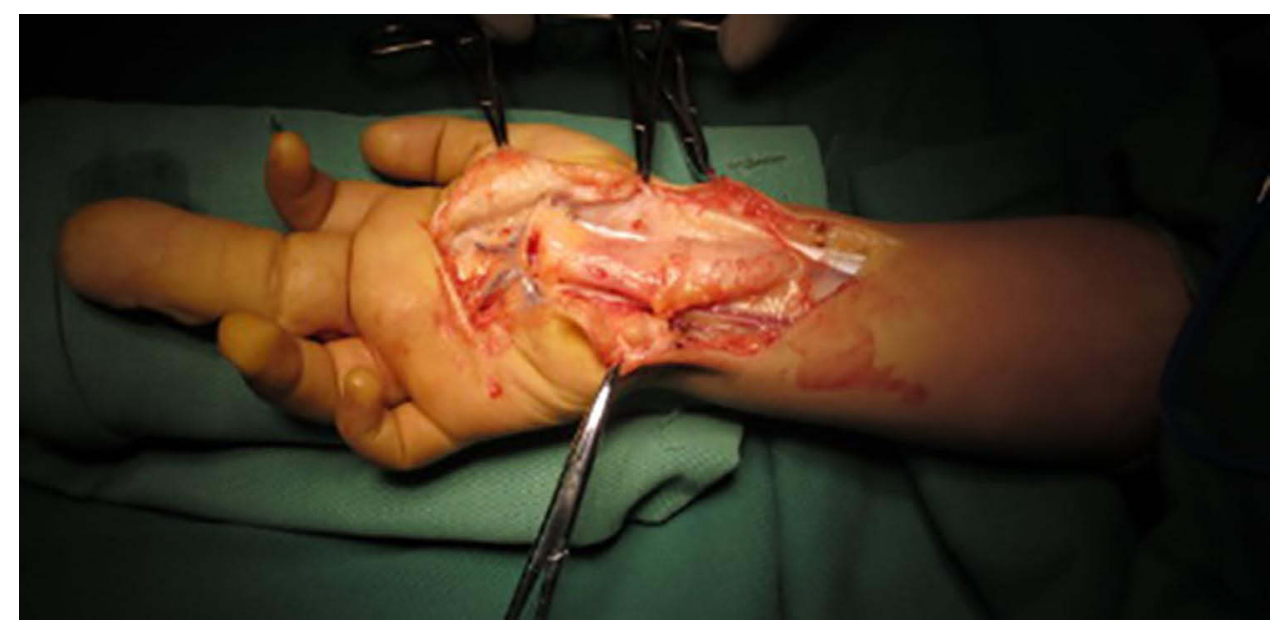

Figure 2 Intraoperative conditions of children with macrodactyly (median nerve thickening, adipose infiltration, and adipose tissue hyperplasia).

(Agilent Technologies, USA). Once the library had been pooled with a specific index, it was hybridized with a biotinlabeled probe, and magnetic beads with streptomycin were used to capture the exons of the gene.

\section{Library Retrieval}

After the library was built, Qubit 2.0 (Life Invitrogen, USA) was used for preliminary quantification, after which NGS3K/Caliper (Perkin Elmer, USA) was used to detect the insert size of the library. Once the insert size met the requirements, the effective concentration of the library was accurately quantified using the quantitative real-time polymerase chain reaction (qPCR) method to ensure the quality of the library.

\section{Sequencing on Machine}

Library quality inspection was performed after PCR amplification, and each sample was loaded into the machine after passing the quality inspection. High-throughput sequencing was performed (Shanghai Genechem Co., Ltd., China) using the Illumina Novaseq 6000 platform (Illumina company, USA). The average sequencing depth of the target area reached $125 \times$ or more, and the coverage of the target area was over $99.97 \%$.

\section{Screening of Mutant Genes}

The genes that met one of the following mutant gene selection criteria were selected:

(1) Mutations with a frequency higher than $1 \%$ in at least one of the four frequency databases (Thousands of Genome Data [1000g_all], ESP6500 Database [esp6500si_all], and gnomAD Data [gnomAD_ALL and gnomAD_EAS]) were excluded in order to remove the diverse sites between individuals and identify rare mutations that may cause disease.

(2) The variations in the coding (exonic) region or splicing site (10 bp up and down) were retained.

(3) The synonymous single-nucleotide polymorphism mutations that were not located in the highly conserved region and not predicted by the software as affecting splicing were excluded. (A site with a grep ++ score $>2$ was considered a highly conserved region; if at least one of the two predicted scores of dbscSNV was greater than 0.6 , it was considered that the mutation would affect splicing.) The small-fragment $(<10 \mathrm{bp})$ non-frameshift InDel mutations in the repeat region were excluded.

(4) Following the evidence from the 2015 American College of Medical Genetics and Genomics, the harmfulness of each mutation site was analyzed; the mutations that met one of the following conditions were retained: (a) the mutation was predicted to be harmful; (b) the mutation was predicted to affect splicing.

\section{Bioinformatics Analysis}

Comprehensive bioinformatics analysis was conducted in four stages: 
(1) The four groups of mutant genes (macrodactyly of finger, macrodactyly of foot, macrodactyly and syndactyly of finger, and macrodactyly and syndactyly of foot) were intersected, and the common mutant genes in the pathological tissues were screened out.

(2) For cases with both tissue specimens and blood specimens, the genes that were only mutated in the tissue were intersected to screen out genes that were only mutated in pathological tissues.

(3) Phenolyzer analysis was conducted on the selected mutant genes. ${ }^{8}$

(4) Sanger sequencing was performed for verification of the screened mutant genes ${ }^{9}$ and to further determine the regions and sites of the mutations.

\section{Immunohistochemical Analysis of Pathological Tissue}

The mutant genes of macrodactyly were screened out based on the results of the WES and verification with Sanger sequencing, and monoclonal antibody immunohistochemical analysis was performed on the protein signaling pathways encoded by the mutant genes in order to observe the protein expression and further verify the mutant genes.

Immunohistochemical analysis of serine-threonine kinase (AKT) monoclonal antibody was performed in the pathological tissue (adipose) to detect the endogenous level of AKT total protein. To observe the expression of AKT protein, the analysis was performed with the first antibody of rabbit anti-AKT monoclonal antibody IgG (Cell Signaling Technology, USA, CST, No:4691T) and the second antibody of goat anti-rabbit antibody IgG labeled with horseradish peroxidase (Cell Signaling Technology, China, Servicebio, No:C030812). The adipose tissue of patients with polydactyly was used as a control group, and the expression intensity of AKT protein was compared. Hematoxylin-eosin staining of adipose cells was also performed.

\section{HE Stains}

The pathological analysis of adipose tissue was carried out by hematoxylin eosin staining. It was fixed in $4 \%$ paraformaldehyde for 48 hours. After being embedded in stone wax, it was prepared into sections with a thickness of $5 \mu \mathrm{M}$. hematoxylin staining, eosin re staining and neutral gum sealing were observed under the microscope.

\section{Results}

\section{Analysis of Mutation Gene Detection in Four Groups of Pathological Tissues}

The number of mutant genes in each group of pathological tissues was: (1) macrodactyly of finger group: 413; (2) macrodactyly of foot group: 1730; (3) macrodactyly and syndactyly of finger group: 340; (4) macrodactyly and syndactyly of foot group: 674 .

Eight mutation genes (PIK3CA, ACTR3C, IST1, MYH8, MUC16, HELZ2, BAGE2, and BAGE5) were identified in the four groups after intersection. The Wayne diagram after intersection is shown in Supplemental Figure 1.

\section{Analysis of Mutation Gene in Pathological Tissue and Peripheral Blood in the Same Patient}

In the cases with both tissue samples and peripheral blood samples, we screened the genes mutated only in the tissue but not in the peripheral blood, and then took the intersection of the genes of 9 samples. PIK3CA is the intersection gene of 9 cases. The Wayne diagram after intersection is shown in Supplemental Figure 2.

\section{Phenolyzer Analysis of Mutation Genes}

Phenolyzer analysis was performed on the 8 mutation genes screened out from the intersection of the four groups. The possible pathogenic genes were then screened according to clinical phenotype. After Phenolyzer analysis, the PIK3CA gene was selected as the seed gene. The upstream and downstream genes are shown in Supplemental Figure 3.

The candidate genes were ranked according to the association between the 8 candidate mutant genes and diseases, as shown in Figure 3. The mutation genes and loci of the WES results are shown in Table 2. 


\section{Score}

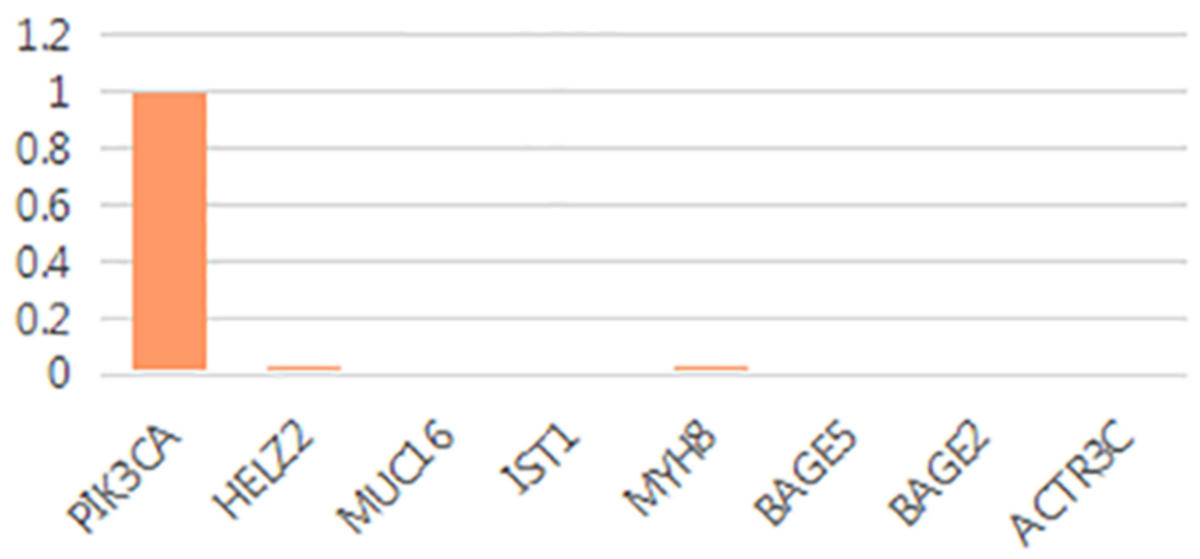

Figure 3 The candidate genes were ranked according to the association between the 8 candidate mutant genes and diseases ( $y$-axis label: relevance score).

\section{Sanger Sequencing Results of the PIK3CA Gene}

Based on the results of WES, the PIK3CA gene was selected as the pathogenic gene, with mutation sites concentrated in exon10 and exon21. Sanger sequencing of exon10 and exon21 of the PIK3CA gene in each sample was then performed. ${ }^{10}$ The sequencing detected the PIK3CA gene mutation in the pathological tissue samples of all 12 patients, and the mutation site and mutation mode identified by Sanger sequencing were the same as those identified by WES. The primer information of the Sanger sequencing of the PIK3CA gene is shown in Supplemental Table 1. The map of mutation sites detected by the Sanger sequencing is shown in Table 3.

\section{Immunohistochemical Analysis of Pathological Tissues}

Hematoxylin-eosin staining revealed that the volume of adipocytes of macrodactyly (finger) was larger than that of polydactyly, but the difference was not significant. Immunohistochemical analysis of the AKT monoclonal antibody IgG revealed that the staining of adipocytes of macrodactyly (finger) was significantly darker than that of polydactyly, suggesting that the expression of AKTprotein was significantly increased. (Figure 4).

Table 2 Sanger Sequencing Mutation Site of PIK3CA Gene in Pathological Tissues of 12 Children

\begin{tabular}{|c|c|c|c|c|}
\hline No. & CytoBand & Exon & Mutant Nucleotides & Amino Acid Change \\
\hline I & $3 q 26.32$ & exon2I & c.A3।40G & p.HI047R \\
\hline 2 & $3 q 26.32$ & exon 10 & c.GI624A & p.E542K \\
\hline 3 & $3 q 26.32$ & exon2I & c.A3।40G & p.HI047R \\
\hline 4 & $3 q 26.32$ & exon2I & c.A3।40G & p.HI047R \\
\hline 5 & $3 q 26.32$ & exon 10 & c.GI624A & p.E542K \\
\hline 6 & $3 q 26.32$ & exon 10 & c.GI633A & p.E545K \\
\hline 7 & $3 q 26.32$ & exon2I & c.G3I45C & p.GI049R \\
\hline 8 & $3 q 26.32$ & exon2I & c.A3।40G & p.HI047R \\
\hline 9 & $3 q 26.32$ & exon2I & c.A3।40G & p.HI047R \\
\hline 10 & $3 q 26.32$ & exon2I & c.A3।40G & p.HI047R \\
\hline II & $3 q 26.32$ & exon2I & c.A3।40G & p.HI047R \\
\hline 12 & $3 q 26.32$ & exon 10 & c.GI633A & p.E545K \\
\hline
\end{tabular}


Table 3 The Results of Sanger Sequencing Gene Detection in 12 Children with Pathological Tissue Were Analyzed

\begin{tabular}{|c|c|c|}
\hline No. & Mutation Site & Genotype \\
\hline $1,3,4,8,9,10,11$ & $\begin{array}{l}\text { PIK3CA } \\
\text { E2I }\end{array}$ & $\begin{array}{l}\text { p. } \mathrm{HI} 047 \mathrm{R} \\
\text { c. } 3 \text { I } 40 \mathrm{~A}>\mathrm{G}\end{array}$ \\
\hline 2,5 & $\begin{array}{l}\text { PIK3CA } \\
\text { EIO }\end{array}$ & $\begin{array}{l}\text { p.E542K } \\
\text { c. } 1624 G>A\end{array}$ \\
\hline 6,12 & $\begin{array}{l}\text { PIK3CA } \\
\text { EI0 }\end{array}$ & $\begin{array}{l}\text { p.E545K } \\
\text { c. } 1633 \mathrm{G}>\mathrm{A}\end{array}$ \\
\hline 7 & $\begin{array}{l}\text { PIK3CA } \\
\text { E2I }\end{array}$ & $\begin{array}{l}\text { P.GI049R } \\
\text { c. } 3145 \mathrm{G}>\mathrm{C}\end{array}$ \\
\hline
\end{tabular}

\section{Discussion}

The exome is the sum of all the exon regions in a species genome and is the most direct embodiment of gene function. Human exome sequences account for about $1 \%$ of all human genome sequences, and $85 \%$ of mutation genes related to human diseases are located in the coding sequences and splicing sites of exons. ${ }^{11}$ The use of WES and high-throughput sequencing has been proven to be highly efficient in the screening of genes for congenital genetic diseases and rare diseases. $^{12}$

A

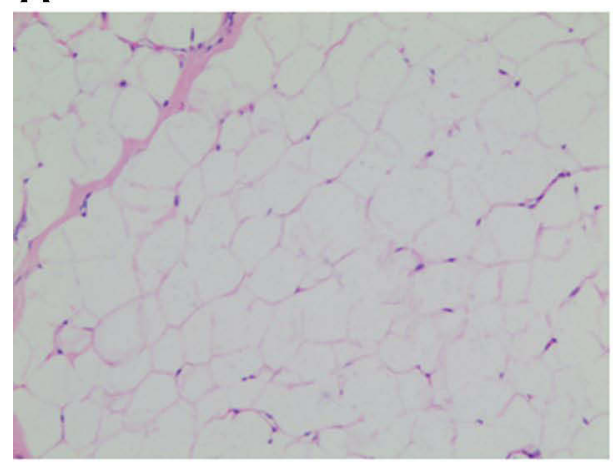

C

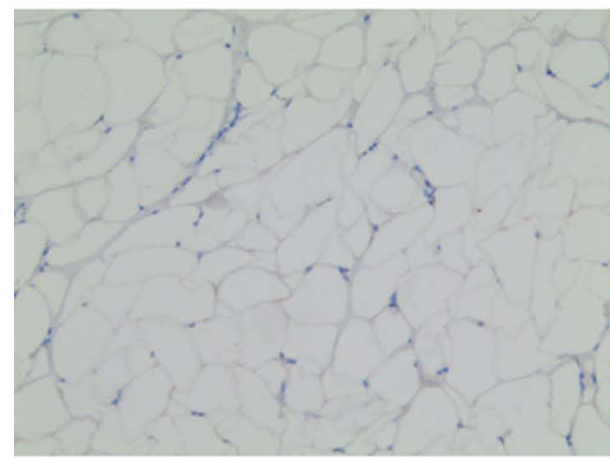

B

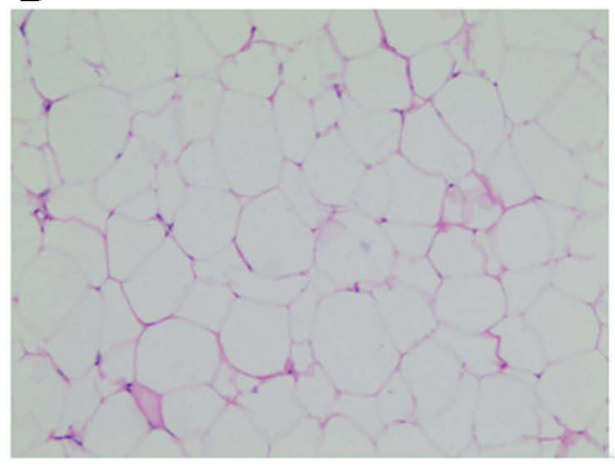

D

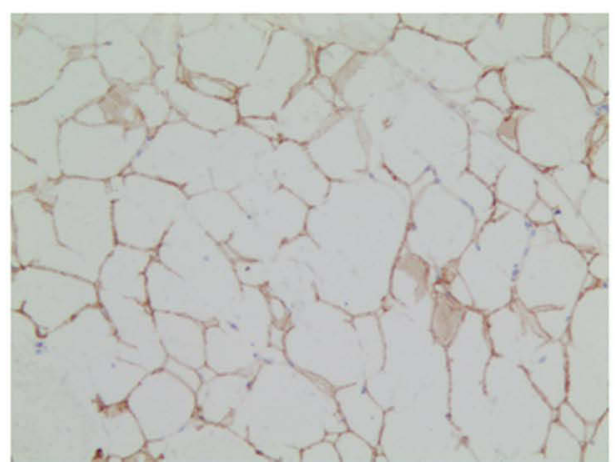

Figure $4 \mathrm{He}$ staining and immunohistochemical staining of adipose tissue (A) He staining of adipocytes in polydactyly children; (B) He staining of adipocytes in children with macrodactyly; (C) Immunohistochemical staining of Akt monoclonal antibody in adipocytes of polydactyly children; (D) Akt monoclonal antibody immunohistochemical staining of adipocytes in children with macrodactyly. $(100 \times)$. 
In the present study, mutant genes in the pathological tissue of four groups (12 cases) of patients with macrodactyly were intersected, and Phenolyzer analysis of the results of the intersection was conducted. This analysis identified the PIK3CA gene as the seed gene that causes the phenotype of macrodactyly. In the comparison of the mutations in the whole exomes of the pathological tissue and the peripheral blood of the patients, only the PIK3CA gene was found to be mutated in the pathological tissues of the 9 patients from whom only tissue samples were taken. In both analyses, the pathogenic gene of macrodactyly was directed to PIK3CA. Immunohistochemistry then revealed that the activity of AKT protein in the adipose tissue of macrodactyly was significantly increased compared with that of polydactyly, and immunofluorescence analysis indicated that AKT was expressed in both the cell membrane and nucleus of adipocytes of macrodactyly. We speculate that the PIK3CA gene mutation leads to the activation of the PI3K-AKT-mTOR signaling pathway, which is the cause of macrodactyly.

The PIK3CA gene, which is located at 3q26.3, is $34 \mathrm{~KB}$ long, contains 21 exons, and encodes 1068 amino acids (a group of amino acids that produces a $124 \mathrm{kD}$ protein) was first detected by Volinia in 1994 by in situ hybridization. ${ }^{13}$ PIK3CA encodes the P110 catalytic subunit of class I phosphatidylinositol-3-kinases (PI3Ks), ie, PI3Kp110a. The main function of PI3K protein is phosphorylation. ${ }^{14}$ The result of the activation of PI3K is that 4,5-diphosphate phosphatidylinositol (PIP2) is catalyzed into the second messenger of 3,4,5-diphosphate phosphatidylinositol (PIP3) with 3 phosphates, leading to the activation of AKT; AKT then transmits the signal to the downstream mTOR to regulate the physiological processes of different cells, such as cell proliferation, metabolism, autophagy, apoptosis, and migration, in order to trigger a series of intracellular signal transductions. ${ }^{13,14}$ This is known as the PI3K-Akt-mTOR signaling pathway participated by PI3K. In the present study, AKT monoclonal antibody immunohistochemical staining was performed on the pathological adipose tissue of patients with macrodactyly and polydactyly. It was observed that AKT protein expression in the adipose tissue of patients with macrodactyly was higher than that of patients with polydactyly, and AKT monoclonal antibody immunofluorescence analysis revealed that AKT was strongly expressed in both the cell membrane and nucleus of adipocytes of macrodactyly. This provided further proof that the mutation of the PIK $3 C A$ gene leads to the activation of PI3K encoded by it and its downstream AKT protein, after which the signal is transmitted to the mTOR, resulting in adipocyte proliferation and the enhancement of metabolism, causing the patients' fingers/toes to develop adipose tissue hyperplasia and nerve-fiber fat infiltration, finally leading to the enlargement of the fingers or toes to form macrodactyly.

Previous studies have reported that the pathogenic mutation of PIK3CA encodes the abnormal P110 $\alpha$-subunit, leading to the continuous activation of the PI3K enzyme; this enhances intracellular signaling conduction, leading to uncontrolled cell proliferation and the formation of tumors, such as breast cancer, endometrial cancer, colon cancer, ovarian cancer, and lung cancer. ${ }^{15-17}$ However, one study has suggested that, as a proto-oncogene, PIK3CA's single pathogenic mutation cannot directly lead to tumors - only the pathogenic mutation that co-occurs with other genes can lead to tumors ${ }^{18}$ - which may be why macrodactyly has biological characteristics that are not completely the same as those of tumors.

Approximately $91 \%$ of the mutation sites of the PIK3CA gene are located in the helical region (p.E542K and $\mathrm{p}$. E545K in exon 10) and kinase region (p.H1047R in exon 21). These mutation sites are all pathogenic and are common hot spots for mutations in tumors. ${ }^{19,20}$ In addition to these tumor mutation sites, p.Arg115Pro, p.Cys420Arg, p. Glu453Lys, and p.His1047Leu, have been reported in the study of the pathogenic gene sites of macrodactyly. ${ }^{7,21-23}$ In the present study, in addition to the previously reported p.E542K, p.E545K, and p.H1047R sites, one patient was found to have a mutation in the p.G1049R (c. $3145 \mathrm{G}>$ C) site, which has been previously reported in megalencephaly-capillary malformation syndrome ${ }^{7}$ but has never been reported in macrodactyly. From the perspective of clinical phenotypes, the patient (case 7) had isolated macrodactyly on the right second and third toes with no other special phenotypes, so this site should be considered a new pathogenic site of macrodactyly.

\section{Conclusion}

Through WES and verification with Sanger sequencing, the pathogenic gene of macrodactyly was identified. PIK3CA gene mutation may have a significant correlation with the pathogenesis of macrodactyly. This provides a theoretical basis for the future study of targeted drug treatment for macrodactyly. 


\section{Ethics Approval and Consent to Participate}

This study was conducted in accordance with the declaration of Helsinki. This study was conducted with approval from the Ethics Committee of Shunyi Teaching Hospital of Capital Medical University. A written informed consent was obtained from all participants.

\section{Consent for Publication}

Consent for publication was obtained from every patient and their legal guardians whose data are included in this manuscript.

\section{Funding}

Beijing Shunyi District Science and Technology Three Fee Projects No. KS201935.

\section{Disclosure}

The authors report no conflicts of interest in this work.

\section{References}

1. Cerrato F, Eberlin KR, Waters P, et al. Presentation and treatment of macrodactyly in children. J Hand Surg Am. 2013;38(11):2112-2123. PMID: 24060511. doi:10.1016/j.jhsa.2013.08.095

2. Marek T, Spinner RJ, Syal A, et al. Strengthening the association of lipomatosis of nerve and nerve-territory overgrowth: a systematic review. J Neurosurg. 2019:1-9. PMID: 30925468. doi:10.3171/2018.12.JNS183050

3. Gluck JS, Ezaki M. Surgical treatment of Macrodactyly. J Hand Surg Am. 2015;40(7):1461-1468. PMID: 26050204. doi:10.1016/j. jhsa.2015.04.017

4. Ezaki M, Beckwith T, Oishi SN. Macrodactyly: decision-making and surgery timing. J Hand Surg Eur Vol. 2019;44(1):32-42. PMID: 30208752. doi:10.1177/1753193418796441

5. Waters PM, Gillespie BT. Ray resection for progressive Macrodactyly of the hand: surgical technique and illustrative cases. $J$ Hand Surg Am. 2016;41(8):e251-6. PMID: 27344071. doi:10.1016/j.jhsa.2016.05.012

6. Ezaki M. Insights into the pathogenesis of macrodactyly. J Hand Surg Eur Vol. 2019;44(1):25-31. PMID: 30086673. doi:10.1177/ 1753193418790928

7. Keppler-Noreuil KM, Rios JJ, Parker VE, et al. PIK3CA-related overgrowth spectrum (PROS): diagnostic and testing eligibility criteria, differential diagnosis, and evaluation. Am J Med Genet A. 2015;167A(2):287-295. PMID: 25557259; PMCID: PMC4480633. doi:10.1002/ajmg.a.36836

8. Yang H, Robinson PN, Wang K. Phenolyzer: phenotype-based prioritization of candidate genes for human diseases. Nat Methods. 2015;12 (9):841-843. PMID: 26192085; PMCID: PMC4718403. doi:10.1038/nmeth.3484

9. Gudbjartsson DF, Helgason H, Gudjonsson SA, et al. Large-scale whole-genome sequencing of the Icelandic population. Nat Genet. 2015;47 (5):435-444. PMID: 25807286. doi:10.1038/ng.3247

10. Rios JJ, Paria N, Burns DK, et al. Somatic gain-of-function mutations in PIK3CA in patients with macrodactyly. Hum Mol Genet. 2013;22 (3):444-451. PMID: 23100325; PMCID: PMC3542862. doi:10.1093/hmg/dds440

11. Van Dijk EL, Auger H, Jaszczyszyn Y, et al. Ten years of next-generation sequencing technology. Trends Genet. 2014;30(9):418-426. PMID: 25108476. doi:10.1016/j.tig.2014.07.001

12. Hucthagowder V, Shenoy A, Corliss M, et al. Utility of clinical high-depth next generation sequencing for somatic variant detection in the PIK3CA-related overgrowth spectrum. Clin Genet. 2017;91(1):79-85. PMID: 27307077. doi:10.1111/cge.12819

13. Volinia S, Hiles I, Ormondroyd E, et al. Molecular cloning, cDNA sequence, and chromosomal localization of the human phosphatidylinositol 3-kinase p110 alpha (PIK3CA) gene. Genomics. 1994;24(3):472-477. PMID: 7713498. doi:10.1006/geno.1994.1655

14. Huang CH, Mandelker D, Schmidt-Kittler O, et al. The structure of a human p110alpha/p85alpha complex elucidates the effects of oncogenic PI3Kalpha mutations. Science. 2007;318(5857):1744-1748. PMID: 18079394. doi:10.1126/science.1150799

15. Xue G, Zippelius A, Wicki A, et al. Integrated Akt/PKB signaling in immunomodulation and its potential role in cancer immunotherapy. $J$ Natl Cancer Inst. 2015;107(7):djv171. PMID: 26071042. doi:10.1093/jnci/djv171

16. Sarris EG, Saif MW, Syrigos KN. The biological role of PI3K pathway in lung cancer. Pharmaceuticals (Basel). 2012;5(11):1236-1264. PMID: 24281308; PMCID: PMC3816662. doi:10.3390/ph5111236

17. Fresno Vara JA, Casado E, de Castro J, et al. PI3K/Akt signalling pathway and cancer. Cancer Treat Rev. 2004;30(2):193-204. PMID: 15023437. doi:10.1016/j.ctrv.2003.07.007

18. Zhao L, Vogt PK. Class I PI3K in oncogenic cellular transformation. Oncogene. 2008;27(41):5486-5496. PMID: 18794883; PMCID: PMC2757120. doi:10.1038/onc.2008.244

19. Samuels Y, Wang Z, Bardelli A, et al. High frequency of mutations of the PIK3CA gene in human cancers. Science. 2004;304(5670):554. PMID: 15016963. doi:10.1126/science. 1096502

20. Campbell IM, Shaw CA, Stankiewicz P, et al. Somatic mosaicism: implications for disease and transmission genetics. Trends Genet. 2015;31 (7):382-392. Erratum in: Trends Genet. 2016 Feb;32(2):138.Erratum in: Trends Genet. 2016 Feb;32(2):138.PMID: 25910407; PMCID: PMC4490042. doi:10.1016/j.tig.2015.03.013

21. Tripolszki K, Knox R, Parker V, et al. Somatic mosaicism of the PIK3CA gene identified in a Hungarian girl with macrodactyly and syndactyly. Eur J Med Genet. 2016;59(4):223-226. PMID: 26851524. doi:10.1016/j.ejmg.2016.02.002 
22. Lackburn PR, Milosevic D, Marek T, et al. PIK3CA mutations in lipomatosis of nerve with or without nerve territory overgrowth. Mod Pathol. 2020;33(3):420-430. PMID: 31481664. doi:10.1038/s41379-019-0354-1

23. Wu J, Tian W, Tian G, et al. An investigation of PIK3CA mutations in isolated macrodactyly. J Hand Surg Eur Vol. 2018;43(7):756-760. PMID: 29661094. doi: $10.1177 / 1753193418770366$

Pharmacogenomics and Personalized Medicine

\section{Publish your work in this journal}

Pharmacogenomics and Personalized Medicine is an international, peer-reviewed, open access journal characterizing the influence of genotype on pharmacology leading to the development of personalized treatment programs and individualized drug selection for improved safety, efficacy and sustainability. This journal is indexed on the American Chemical Society's Chemical Abstracts Service (CAS). The manuscript management system is completely online and includes a very quick and fair peer-review system, which is all easy to use. Visit http://www. dovepress.com/testimonials.php to read real quotes from published authors.

Submit your manuscript here: https://www.dovepress.com/pharmacogenomics-and-personalized-medicine-journal 